\title{
LncRNA HULC shRNA disinhibits miR-377-5p to suppress the growth and invasion of hepatocellular carcinoma in vitro and hepatocarcinogenesis in vivo
}

\author{
Chunxiao Yan, Shutang Wei, Dazheng Han, Liping Wu, Lixia Tan, Hangyu Wang, Yong Dong, Jing Hua, \\ Wenyi Yang \\ Department of Gastroenterology, The First Affiliated Hospital of Henan University, Kaifeng, China \\ Contributions: (I) Conception and design: C Yan, S Wei; (II) Administrative support: W Yang; (III) Provision of study materials or patients: C Yan, S \\ Wei, D Han, L Wu; (IV) Collection and assembly of data: L Tan, H Wang, Y Dong, J Hua; (V) Data analysis and interpretation: C Yan, S Wei, D \\ Han, L Wu; (VI) Manuscript writing: All authors; (VII) Final approval of manuscript: All authors. \\ Correspondence to: Wenyi Yang. Department of Gastroenterology, The First Affiliated Hospital of Henan University, No. 357 Ximen Street, Longting \\ District, Kaifeng 475001, China. Email: baoca4621210@163.com.
}

Background: Aberrant expression of up-regulated long non-coding RNA [LncRNA highly upregulated in liver cancer (HULC)] has been observed to play an important regulatory role in the development of multiple human diseases. However, the molecular mechanism underlying the role of HULC and miR-377-5p in HCC needs to be urgently explored.

Methods: The mRNA and protein expression levels of HULC were detected by quantitative real-time polymerase chain reaction (qRT-PCR) and western blot in hepatocellular carcinoma (HCC) cell line HB611, HepG2 and H22, respectively. HULC-shRNA was transfected into HepG-2 cells, which were randomly divided into the control, shRNA-NC, and sh-HULC groups. The correlation between HULC and miR377-5p was analyzed by performing a luciferase reporter assay. The targeting relationship between miR$377-5 \mathrm{p}$ and hypoxia-inhibitory factor- $1 \alpha(\mathrm{HIF}-1 \alpha)$ was also investigated using a luciferase reporter assay. Sh-HULC and miR-377-5p inhibitors were transfected either alone or together into HepG2 cells, and which were divided into the control group, the sh-HULC group, the miR-377-5p inhibitor, and the sh-HULC + inhibitor group for subsequent experiments. HepG2 cell proliferation and invasion were measured by 5-Ethynyl-2-Deoxyuridine (EdU) staining and Transwell invasion assay, respectively. Western plot was carried out to detect the protein expression levels of Ki67, PCNA, E-cadherin, and N-cadherin. Tumor xenograft mouse models were established to confirm the effect of HULC down-regulation on the development of HCC in vivo.

Results: The mRNA and protein expression levels of HULC were markedly increased, whereas the mRNA expression levels of miR-377-5p were decreased in HCC cell lines. HepG2 cell proliferation and invasion were suppressed in the Sh-HULC group, while miR-377-5p showed the opposite. Further experiments exhibited that miR-377-5p was targeted by HULC, and an negative correlation between HULC and miR377-5p was observed. Importantly, the in vivo experiments indicated that down-regulation of HULC could inhibit tumor growth. Taken together, our research demonstrated that down-regulation of HULC plays an anti-cancer role through restrainingHepG2 cell proliferation and invasion.

Conclusions: In summary, our in vitro and in vivo findings confirmed HULC to play a role in the progression of HCC, with the underlying mechanism possibly involving the miR-377-5p/HIF-1 $\alpha$ pathway.

Keywords: Highly upregulated in liver cancer (HULC); hepatocellular carcinoma (HCC); miR-377-5p; invasion, apoptosis, hepatocarcinogenesis

Submitted Jun 28, 2020. Accepted for publication Sep 18, 2020.

doi: 10.21037/atm-20-5556

View this article at: http://dx.doi.org/10.21037/atm-20-5556 


\section{Introduction}

Hepatocellular carcinoma (HCC) ranks third deadliest of all cancers globally, accounting for more than 600,000 fatalities annually (1). HCC possesses a strong ability to invade hepatic vessels (2). The molecular mechanism of hepatocarcinogenesis is complex and multi-staged, which may be related to genetic and epigenetic factors (3). HCC remains an intractable problem worldwide.

Recently, a number of long non-coding RNAs (lncRNAs) have been discovered to carry out pivotal roles in various biological functions and disease processes, including those of cancer (4). LncRNAs may act as miRNA sponges. In $\mathrm{HCC}$, the expressions of many lncRNAs are dysregulated, including those of maternally expressed gene-3 (MEG-3), metastasis-associated lung adenocarcinoma transcript 1 (MALAT1), highly upregulated in liver cancer (HULC), HOX transcript antisense RNA (HOTAIR), and H19, which is associated with tumorigenesis, metastasis, prognosis, and diagnosis (5). HULC functions as an oncogene in several cancers including ovarian cancer (6), colorectal cancer (7), pancreatic cancer (8), and prostate cancer (9). Therefore, the molecular mechanism underlying the role of HULC in HCC needs to be urgently explored.

Small non-coding RNAs, known as microRNAs (miRNAs), are an important mechanism of posttranscriptional gene regulation, which affects cell differentiation, proliferation, metabolism, apoptosis, cancer, and other processes (10). Various miRNAs are abnormally expressed in HCC, some of which are HCCspecific miRNAs (11). MiR-377 has been demonstrated to regulate the proliferation and invasion of HCC cells $(12,13)$. However, the molecular mechanism underlying the role of $\mathrm{HULC} / \mathrm{miR}-377-5 \mathrm{p} / \mathrm{HIF}-1 \alpha$ in HCC needs to be urgently explored. In the present study, we aimed to examine the $\mathrm{HULC} / \mathrm{miR}-377-5 \mathrm{p} / \mathrm{HIF}-1 \alpha$ axis in HCC.

We present the following article in accordance with the ARRIVE reporting checklist (available at http://dx.doi. org/10.21037/atm-20-5556).

\section{Methods}

\section{Cell culture}

Human normal liver cell line L-02was obtained from the BeNa Culture Collection Biological Technology Co., Ltd. (Beijing, China), along with HCC cell lines HB611, HepG2, and H22. Eagle's Minimum Essential Medium (EMEM; Gibco) supplemented with FBS (Life
Technologies, Grand Island, USA) and $100 \mathrm{U} / \mathrm{mL}$ penicillin and $100 \mathrm{mg} / \mathrm{mL}$ streptomycin was used to culture HCC cell lines, which were incubated at $37{ }^{\circ} \mathrm{C}$ in $95 \% \mathrm{O}_{2}$ with $5 \% \mathrm{CO}_{2}$. Upon reaching $90 \%$ confluency, the cells were collected, and the medium was changed every 48-72 hours.

\section{Quantitative real-time polymerase chain reaction (qRT- PCR)}

Trizol reagent (Invitrogen, Carlsbad, CA, USA) was used to extract total RNA from HepG2 tumor tissue cells. Then, $200 \mathrm{ng}$ of extracted RNA was used for reverse transcription into cDNA using a PrimeScript ${ }^{\mathrm{TM}}$ RT reagent Kit (TaKaRa). The SYBR Premix Ex Taq $^{\mathrm{TM}}$ II (TaKaRa) and a LightCycler 480 Real-Time PCR system (Roche, Shanghai, China) were utilized for qRT-PCR. The $2^{-\triangle \Delta C T}$ method was employed to analyze the candidate genes' relative fold changes. The primers for HULC were as follows: (forward, 5'-CTGGCAATAAACTAAGCA-3' and reverse 5'-CAACATAATTCAGGGAGAA-3'. Primer for miR-377-5p (forward, 5'-GTTTGTT TTAGGGTTATAGAAGTTGG-3' and reverse 5 'ATATAACCRTATTCAATCCAACCTAC'). Primer for shRNA U6 (5'-CTCGCTTCGGCAGCACA-3' and reverse 5'-AACGCTTCACGAATTTGCGT-3'). The primers were synthesized by SangonBioteh (Shanghai, China).

\section{Cell transfection}

HepG2 cells were cultured in 6-well plates at a density of $1 \times 10^{4}$ cells $/ \mathrm{mL}$. After incubation for 24 hours, HepG2 cell lines were subjected to transfection with $50 \mathrm{nM}$ shRNANC, Sh-HULC, mimic-NC, miR-377-5p mimic, or miR$377-5$ p inhibitor using Lipofectamine ${ }^{\circledR} 3000$ (Invitrogen) in line with the manufacturer's protocol. To target HULC, pGPU6/Neo plasmid (GenePharma, Shanghai, China) was cloned from short-hairpin RNA (sh-RNA) oligonucleotides and the corresponding negative controls (14). ShRNA-NC, Sh-HULC, mimic-NC, miR-377-5p mimic, and miR-377$5 \mathrm{p}$ inhibitors were obtained from Shanghai GenePharma Inc. (Shanghai, China). The scrambled control shRNA (shRNA-NC) and miRNA control (mimic-NC) were used as controls. pcDNA ${ }^{\mathrm{TM}} 3.1$ was bought from Invitrogen (USA). The HIF- $1 \alpha$ pcDNA vector was constructed to overexpress HIF-1 $\alpha$ and transfected into HepG2 cells. The cells were randomly divided into three groups, the control group, the pcDNA group, and the pcDNA-HIF-1 $\alpha$ group. 
RT-PCR and Western blot were used to determine whether the overexpression was successful.

\section{Western blot}

HepG2 cells were harvested from each group, and the total protein was extracted using $1 \mathrm{~mL}$ ice-cold RIPA buffer containing $2 \mathrm{mM}$ phenylmethylsulfonyl fluoride (PMSF) and cocktail. A BCA kit, purchased from Beyotime Institute of Biotechnology (China), was used to measure the protein concentration and. Subsequently, sodium dodecyl sulfate polyacrylamide gel electrophoresis (SDS-PAGE) was carried out to separate $20 \mu \mathrm{g}$ of proteins. After separation, the proteins were transferred to polyvinylidene difluoride (PVDF) membranes. The membranes were sealed with $5 \%$ skimmed dry milk for 1 hour at room temperature, before the blocking solution was discarded and primary antibodies were added for incubation at $4{ }^{\circ} \mathrm{C}$ overnight. The primary antibodies were: rabbit $\beta$-actin $(1: 1,000, \# 4970$, Cell Signaling), rabbit anti-Ki67 (1:1,000, ab243878, Abcam), rabbit anti-PCNA (1:1,000, ab92552, Abcam), rabbit anti-E-cadherin (1:10,000, ab40772, Abcam), rabbit anti-N-cadherin (1:5,000, ab76011, Abcam), and rabbit anti-HIF- $\alpha$ (1:500, ab51608, Abcam). After that, goat antirabbit IgG horseradish peroxidase (HRP)-conjugated secondary antibody was added to the membranes, which were incubated for $60 \mathrm{~min}$ at $37^{\circ} \mathrm{C}$. Enhanced HRP (Pierce, Rockford, IL, USA) was employed to visualize the signals. An automatic digital gel image analysis system (Bio-Rad CFX-96, Bio-Rad, CA, USA) was used to determine and analyze the band densities.

\section{Luciferase reporter assay}

We constructed 3'UTR-HULC as previously described (15). PCR was performed to amplify the 3'UTR of HULC mRNA containing the predicted miR-377-5p binding site or mutant binding site, before its insertion into the pmirGLO dual luciferase expression vector (Promega, Madison, WI, USA). Using Lipofectamine 3000, HepG2 cells were transfected with miR-377-5p mimic and then cotransfected with HULC wild-type (HULC-Wt) or HULC mutation-type (HULC-Mut). TargetScan was used to predict the sequences of HULC-Wt and HULC-Mut, and the reporter plasmids were synthesized by GenePharma. Using Lipofectamine 3000, HepG2 cells were transfected with miR-377-5p mimic and then co-transfected with HIF$1 \alpha$ wild-type (HIF-1 $\alpha$-Wt) or HIF-1 $\alpha$ mutation-type (HIF-
$1 \alpha$-Mut). TargetScan was used to predict the sequences of HIF- $1 \alpha-W t$ and HIF-1 $\alpha$-Mut (Figure S1), and the reporter plasmids were synthesized by GenePharma. After 48 hours of transfection, a dual-luciferase reporter assay system (Promega Corporation, Madison, WI, USA) was used for analysis, in line with the instructions of the manufacturer. Data were normalized to Renilla activity.

\section{Transwell invasion assay}

HepG2 cells transfected with Sh-HULC or miR-377-5p inhibitor or co-transfected with Sh-HULC and miR-377-5p inhibitor were cultured in a 24-well chamber. A Transwell invasion assay was carried out to analyze the HepG2 cells' invasion capacity. Briefly, the cells were placed in the upper chamber in for culture with Dulbecco's Modified Eagle Medium (DMEM) supplemented with 0.1\% FBS. DMEM with $10 \%$ FBS was added to fill the lower chamber. Following 24 hours' incubation, $95 \%$ ethanol was used to fix the bottom cells, which were then stained with hematoxylin. Cells were selected four randomly of microscopic fields. The images were observed and counted with the assistance of an Olympus DX51 fluorescence microscope (Olympus, Tokyo, Japan).

\section{5-Ethynyl-2-Deoxyuridine (EdU)staining for cell proliferation}

HepG2 cells from all of the groups underwent culture in a 24-well chamber. A Cell Light ${ }^{\mathrm{TM}}$ EdU Apollo ${ }^{\circledR} 488$ In Vitro Imaging kit was employed to perform a 5-ethynyl2'-deoxyuridine (EdU) incorporation assay to analyze cell proliferation, in line with the manufacturer's protocol (RiboBio). Images of cells in four randomly selected microscopic fields were observed and counted using an Olympus DX51 fluorescence microscope (Olympus, Tokyo, Japan).

\section{Wound bealing assay}

HepG2 cells were seeded in 6-well plates at a density of $2 \times 10^{5}$ cells/well. Until they reached confluence of $90 \%$, wound gaps were carefully created with a sterile $200 \mu \mathrm{L}$ pipette tip. The cells were washed twice with PBS to remove debris. The remaining cells were washed with cold PBS three times gently. A proliferation inhibitor mitomycin C $(10 \mu \mathrm{g} / \mathrm{mL})$ was added to the cell culture medium to inhibit cell replication. Then the cells were cultured in 
complete medium for $24 \mathrm{~h}$. Same marked areas were rephotographed and calculated using Image J software. Images were acquired with a light microscope (Olympus, Tokyo, Japan) at 100× magnification.

\section{Animal experimental protocols}

Female four-week-old BALB/c nude mice were supplied by Beijing Huafukang Bioscience Co. Inc. (Beijing, China). All animal experiments in this study received approval from the Committee for Animal Experiments of the First Affiliated Hospital of Henan University. This study also conformed to the NIH guidelines. The mice were housed under a 12-hour light/dark cycle in a controlled environment at $25 \pm 3{ }^{\circ} \mathrm{C}$ with $60 \%$ humidity. The mice were given free access to water. The mice were equally divided into 2 random groups: the control group (HepG2 cells transfected with ShRNA-NC) and the Sh-HULC group (HepG2 cells transfected with Sh-HULC). The mice were administered HepG2 cells via subcutaneous injection into their flanks. After 30 days, the mice were sacrificed and the tumors were weighed.

\section{Immunobistochemistry}

The tumor tissues from each group of mice were fixed with $4 \%$ paraformaldehyde. After 24 hours, the tissues were embedded in paraffin, and sectioned to a thickness of about $4 \mu \mathrm{m}$. Xylene was used to separate the sections before they were rehydrated using gradient ethanol. Then, antigen extraction was carried out with $10 \mathrm{mM}$ citric acid buffer. The tissue sections were subsequently incubated in $3 \%$ $\mathrm{H}_{2} \mathrm{O}_{2}$ for 10 minutes, before sealing at room temperature for 1 hour. Following that, the sections were subjected to overnight incubation with rabbit anti-Ki67 (1:100, ab243878, Abcam) and rabbit anti-E-cadherin (1:500, ab40772, Abcam). The corresponding second antibody was incubated at room temperature for 1 hour. Finally, the images were observed with an Olympus DX51 fluorescence microscope (Olympus, Tokyo, Japan), and the data were analyzed with Image-Pro Plus 6.0 (Media Cybernetics, USA).

\section{Bioinformatics data set}

The interaction between miR-377-5p and HULC was predicted with miRnada(http://www.microrna.org) and TargetScan (http://www.targetscan.org).

\section{Statistical analysis}

Each experiment in this study was carried out three times. The data were presented as mean \pm SD. IBM SPSS Statistics 25.0. (IBM, Armonk, USA) was used for statistical analysis of all data. Student's $t$-test was used to analyze differences between the two groups. The significance of differences between treatment groups was studied using one-way analysis of variance (ANOVA). A P value of $<0.05$ was considered to show statistical significance.

\section{Results}

\section{HULC was abnormally expressed in HCC cells}

To study the effect of HULC in HCC progression, the expression levels of HULC were detected in normal liver cell L02 and HCC cell lines HB611, HepG2, and H22. As shown in Figure $1 A, B$, the results showed that compared with normal L02 cells, the mRNA and protein expression levels of HULC were significantly increased in HB611 cells $(\mathrm{P}<0.05)$, H22 cells $(\mathrm{P}<0.05)$, and especially HepG2 cells $(\mathrm{P}<0.01)$. In comparison to the shRNA-NC group, the mRNA and protein expression levels of HULC were markedly decreased in the HULC shRNA transfection (shHULC) group, while the shRNA-NC group displayed no significant changes compared with the control group (Figure 1C,D).

\section{Sh-HULC inbibited HepG2 cell proliferation and invasion}

To obtain a deeper understanding of HULC's role in HCC, the effect of HULC on the proliferation and invasion of HepG2 cells was examined. As shown in Figure 2A, there were fewer numbers of EdU-positive cells (red) in the sh-HULC group in comparison to the control group $(\mathrm{P}<0.05)$. Besides, the protein expression levels of Ki67 and PCNA were reduced by Sh-HULC compared with the control group (Figure $2 B, \mathrm{P}<0.05$ ). Additionally, HepG2 cells invasion ability was restrained in the sh-HULC group in comparison to the control group (Figure $2 C$, $\mathrm{P}<0.05)$. HepG2 cells migration ability was restrained in the sh-HULC group in comparison to the control group (Figure 2D,E, $\mathrm{P}<0.05$ ). Interestingly, the Sh-HULC group had increased protein expression levels of $\mathrm{E}$-cadherin while those of $\mathrm{N}$-cadherin were down-regulated in contrast with the control group (Figure $2 F, G, \mathrm{P}<0.05$ ). These findings suggested that down-regulation of HULC might inhibit the proliferation and invasion of HepG2 cells. 
A

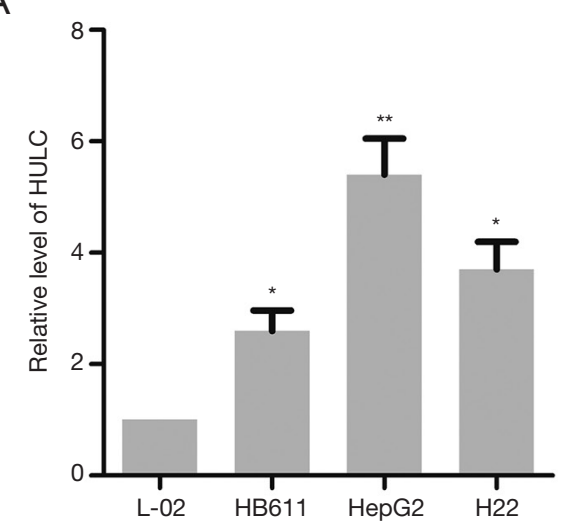

B
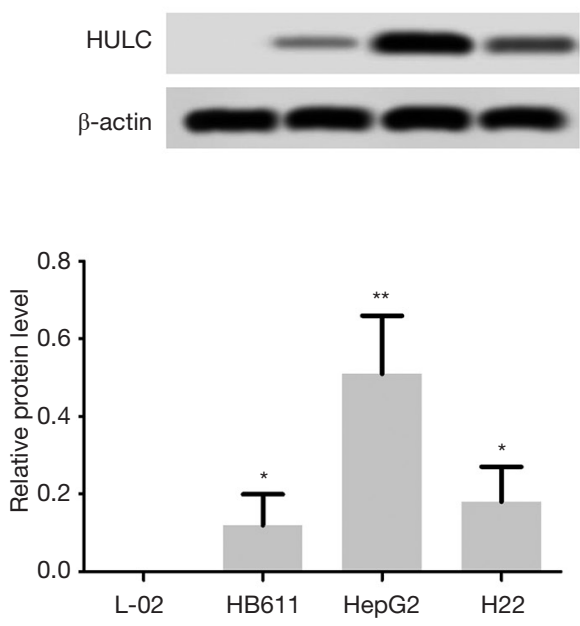

C

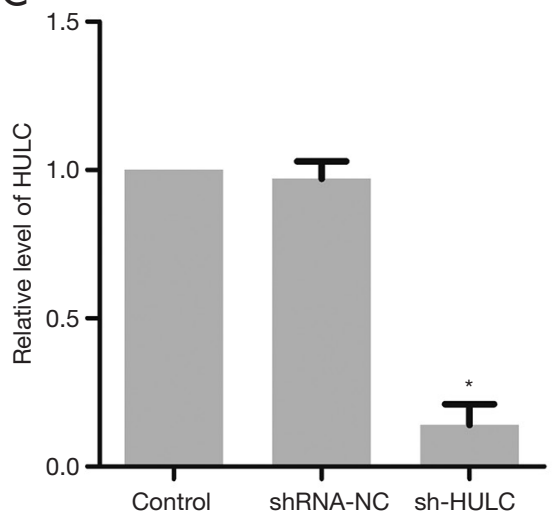

$\mathrm{D}$
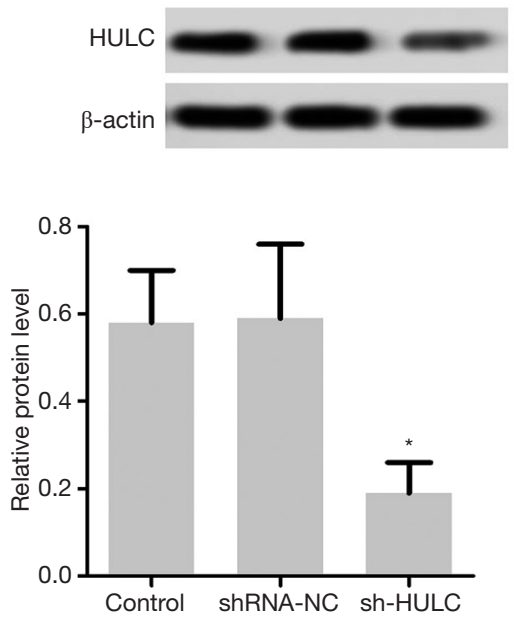

Figure 1 The expression of HULC was detected in HCC cells. (A) The expression of HULC was detected by qRT-PCR in liver normal cell line L02 and HCC cell lines HepG2, HB611, and H22. (B) The expression of HULC was detected by western blot in liver normal cell line L02 and HCC cell lines HepG2, HB611, and H22. *, P<0.05, ${ }^{* *}, \mathrm{P}<0.01$, compared with the L02 group. (C) HULC expression was detected by qRT-PCR in HepG2 cells after transfection with HULC shRNA and shRNA-NC. (D) HULC expression was detected by western blot in HepG2 cells after transfection with HULC shRNA and shRNA-NC. *, $\mathrm{P}<0.05$, compared with the control group. HULC, highly upregulated in liver cancer; HCC, hepatocellular carcinoma; qRT-PCR, quantitative real-time polymerase chain reaction.

\section{miR-377-5p was repressed by HULC}

To study the function of miR-377-5p in HCC progression, the expression levels of miR-377-5p were detected in normal liver cell L02 and HCC cell lines HB611, HepG2, and H22. As shown in Figure 3A, the levels of miR-3775p expression in HB611 cells $(\mathrm{P}<0.05), \mathrm{H} 22$ cells $(\mathrm{P}<0.05)$, and especially HepG2 cells $(\mathrm{P}<0.01)$, were markedly lower than in normal L02 cells. As expected, the levels of miR$377-5 \mathrm{p}$ were up-regulated in the sh-HULC group in contrast with the control group (Figure $3 B, \mathrm{P}<0.05$ ). As shown in Figure 3C, the 3'UTR of HULC-Wt contains elements complementary to miR-377-5p regions. miR-377$5 \mathrm{p}$ expression was markedly elevated by $\mathrm{miR}-377 \mathrm{mimic}$ (Figure 3D, $\mathrm{P}<0.05$ ), while the mimic-NC showed no difference compared with the control group. As described in Figure 3E, cells co-transfected with HULC-Wt and miR-377-5p displayed reduced luciferase activity $(\mathrm{P}<0.05)$; however little effect was observed in cells co-transfected 
A
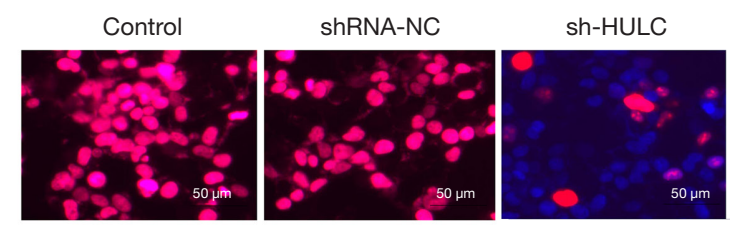

B
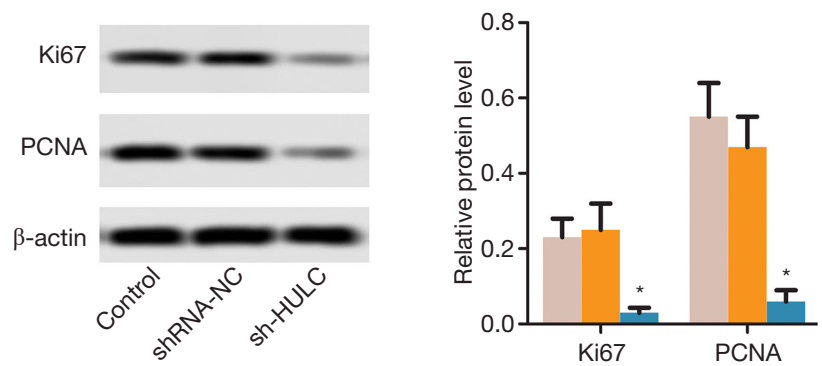

C

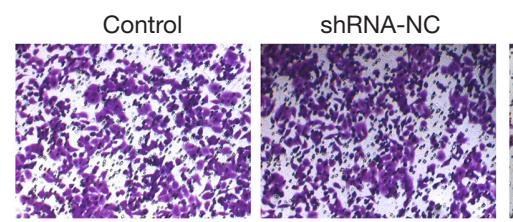

sh-HULC

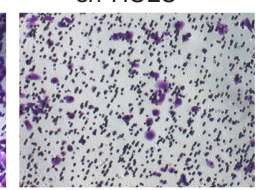

sh-HULC

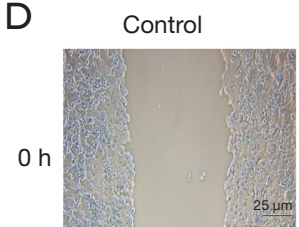

shRNA-NC
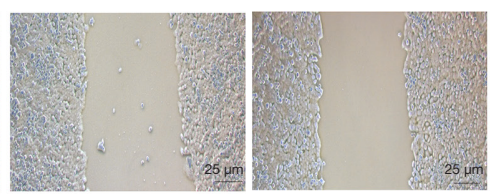

$24 \mathrm{~h}$
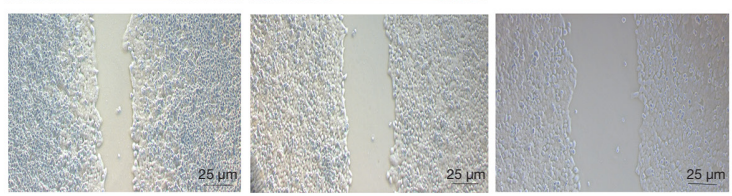

E

Control

ShRNA-NC

sh-HULC
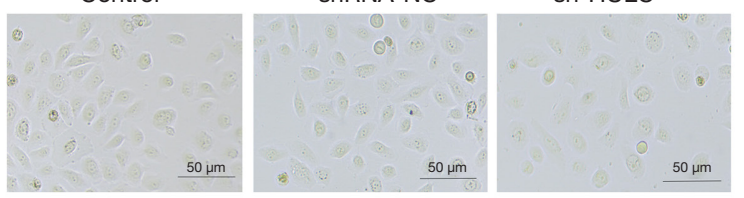

F
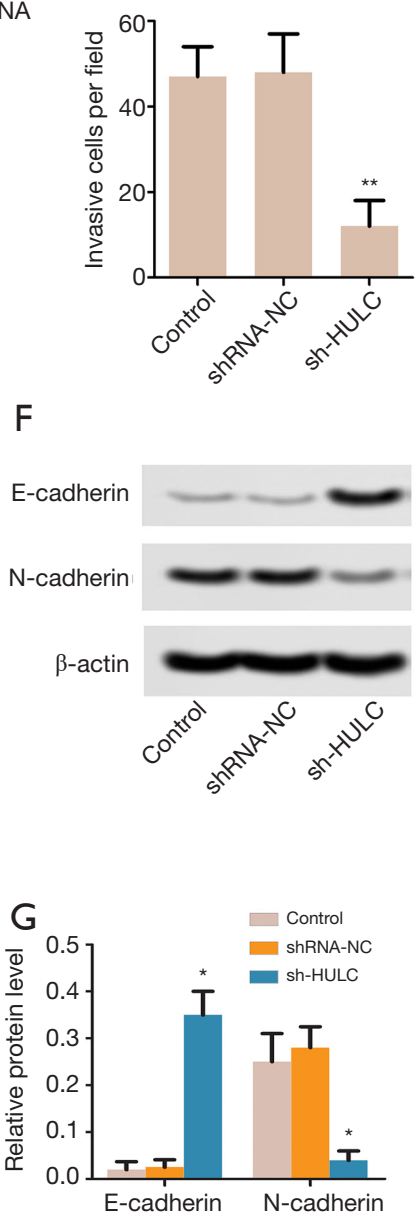

Figure 2 Sh-HULC inhibited the proliferation and invasion of HepG2 cells. (A) The proliferation of HepG2 cells was detected by EdU staining. The EdU-positive (+) cells were quantified. (B) The relative protein levels of Ki67 and PCNA were detected by western blot. Quantitation of signal intensities was performed using ImageJ software. (C) The invasion ability of HepG2 cells was detected by Transwell assay. HepG2 cells were stained with hematoxylin. (Magnification 40x). (D) The migration ability of HepG2 cells was detected by wound healing assay. (E) Epithelial mesenchymal transition (EMT) assay. (F,G) The relative protein levels of E-cadherin and N-cadherin were detected by western blot. The signal intensities were quantitated using Image $\mathrm{J}$ software. *, $\mathrm{P}<0.05$, compared with the control group; **, $\mathrm{P}<0.01$, compared with the control group. HULC, highly up-regulated in liver cancer. 

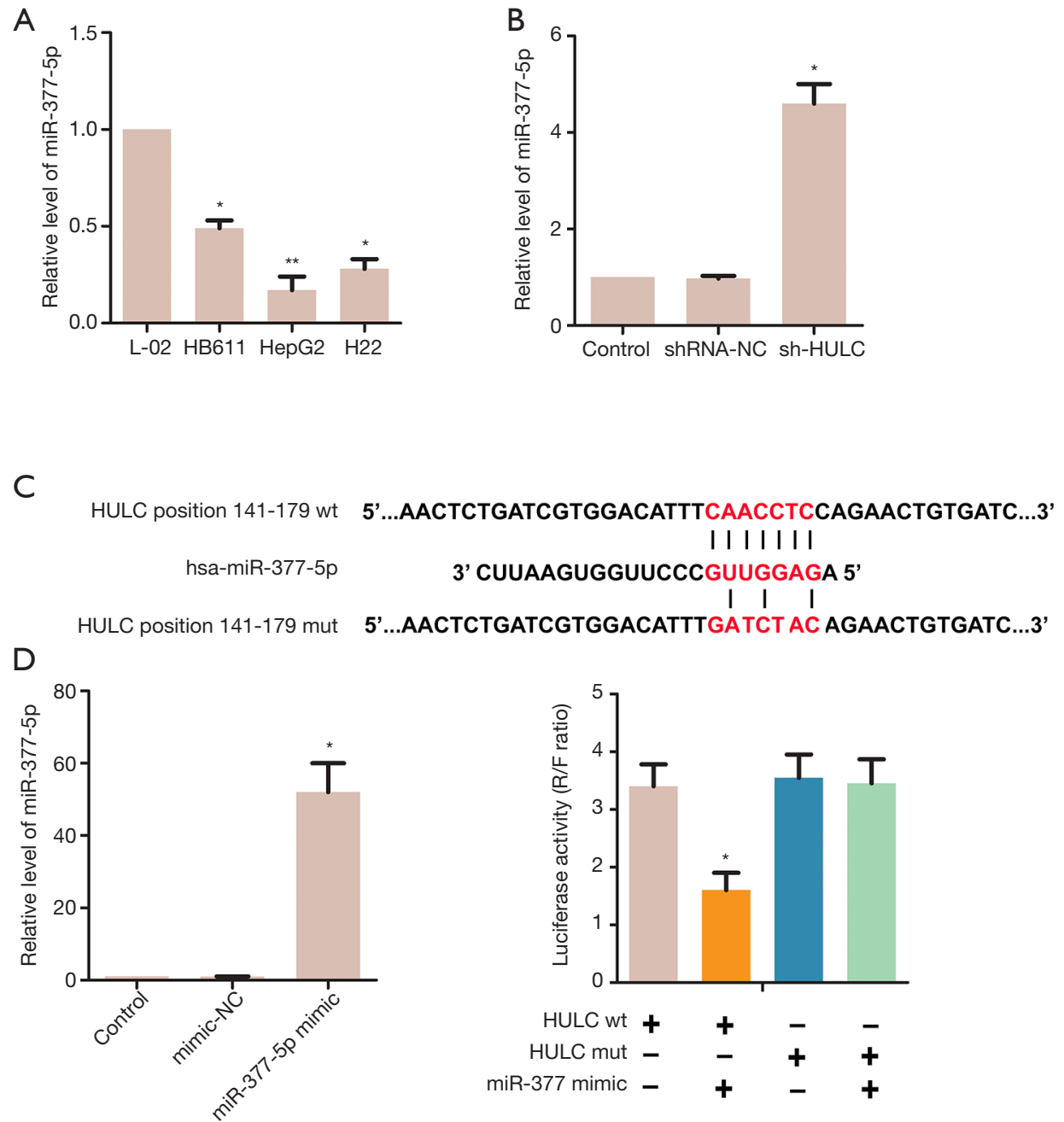

Figure 3 HULC could directly interact with miR-377-5p in HepG2 cells. (A) The expression levels of miR-377-5p were detected by qRTPCR in L02 cells and HCC cell lines HepG2, HB611 and H22. *, P<0.05, **, P<0.01, compared with the L02 group. (B) The expression levels ofmiR-377-5p were detected in HepG2 cells after transfection with HULC shRNA and shRNA-NC. *, P<0.05, compared with the control group. (C) The binding sites between HULC and miR-377-5p were predicted. (D) The mRNA level of miR-377-5p in HepG2 cells was detected by qRT-PCR after miR-377 mimic or mimic-NC. (E) Luciferase activity was detected by luciferase reporter assay. HepG2 cells were co-transfected with either $50 \mathrm{nM}$ miR-377-5p mimics or NC oligos and $200 \mathrm{ng}$ of pmirGLO-HULC-wt or pmirGLO-HULC-mut using Lipofectamine 3000. *, $\mathrm{P}<0.05$, compared with the HULC-wt group. HULC, highly upregulated in liver cancer; HCC, hepatocellular carcinoma; qRT-PCR, quantitative real-time polymerase chain reaction.

with HULC-Mut and miR-377-5p $(\mathrm{P}>0.05)$. These results demonstrated that miR-377-5p was targeted by HULC.

\section{Sh-HULC suppressed proliferation and invasion via derepression of miR-377-5p}

Down-regulation of HULC decreased numbers of the EdU-positive cells $(\mathrm{P}<0.05)$ obviously in comparison to the control group $(\mathrm{P}<0.05)$, while miR-377-5p inhibitor had the reverse effect $(\mathrm{P}<0.05)$. The numbers of the EdUpositive cells were decreased in the sh-HULC + inhibitor group compared with miR-377-5p inhibitor (Figure 4A, $\mathrm{P}<0.05)$. The protein expressive of Ki67 and PCNA were up-regulated in miR-377-5p inhibitor group compared with control group, but the protein expressive of Ki67 and PCNA were down-regulated in the sh-HULC + inhibitor 
A
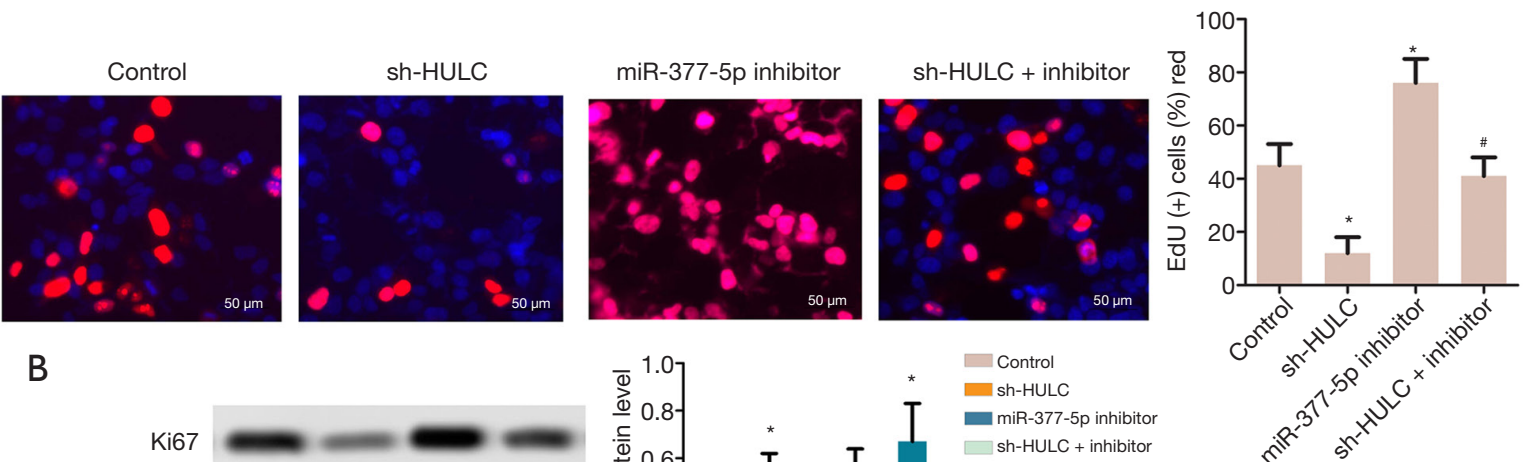

B

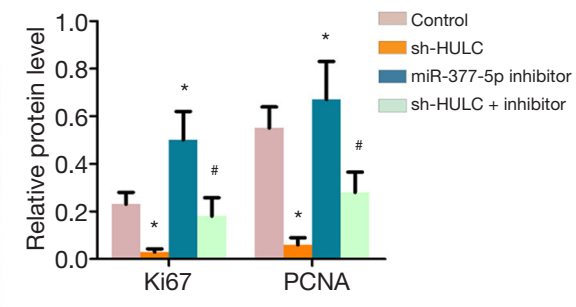

C
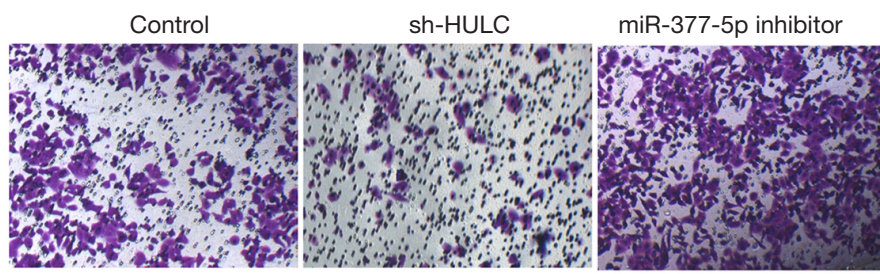

sh-HULC + inhibitor

$\mathrm{D}$

Control
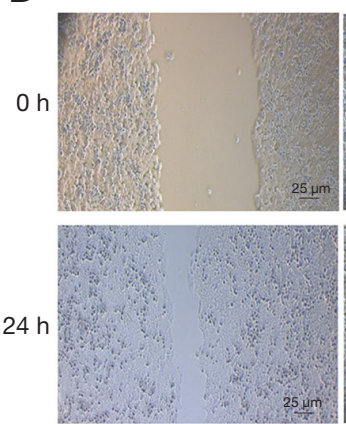

sh-HULC
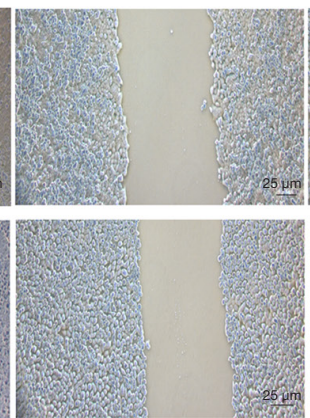

E

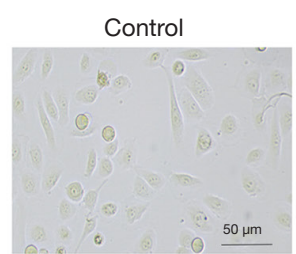

sh-HULC

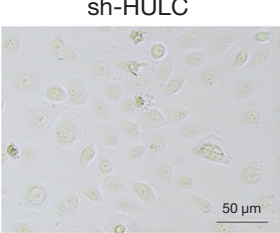

miR-377-5p inhibitor
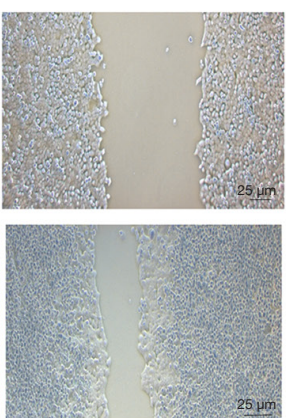

miR-377-5p inhibitor

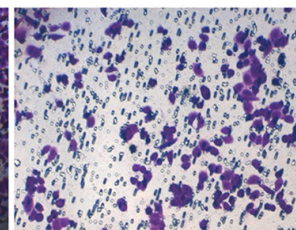

sh-HULC + inhibitor
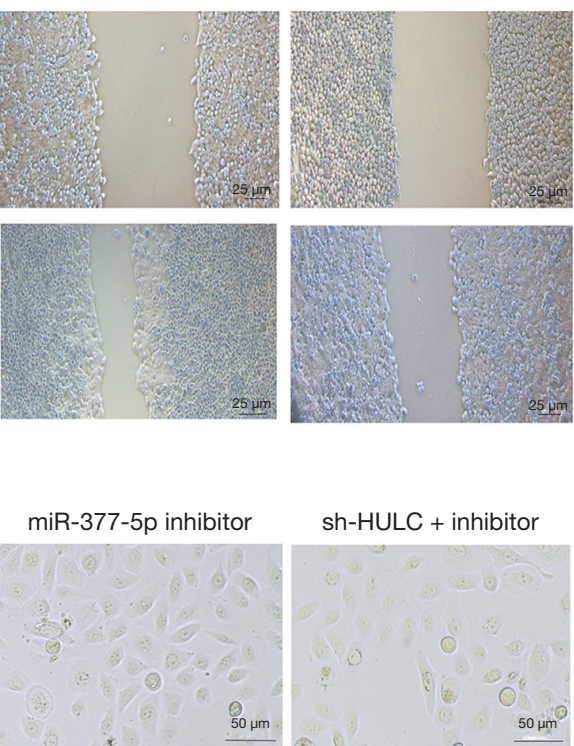

sh-HULC + inhibitor

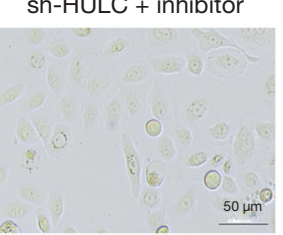

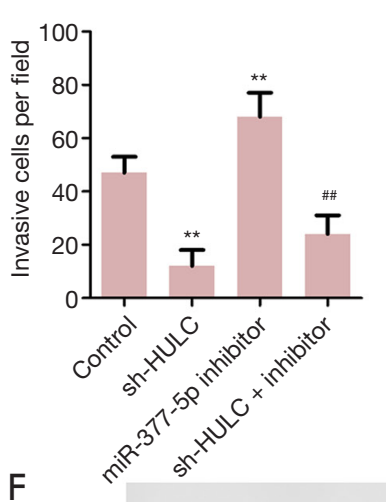
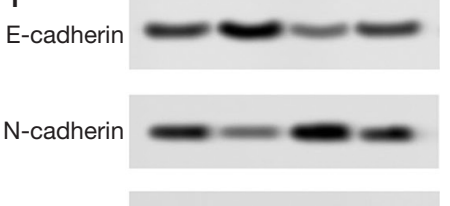

$\beta$-actin
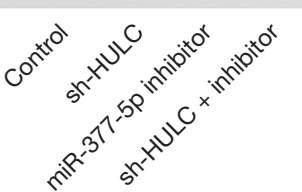

G $\quad$ control

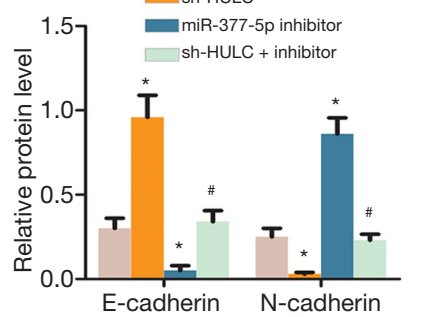

Figure 4 Sh-HULC suppressed the invasion and migration abilities of HepG2 cells. Cells were divided into four groups: the control group, the shHULC group, the miR-377 inhibitor group, and the sh-HULC + inhibitor group. (A) The proliferation of HepG2 cells was detected by EdU staining. The EdU-positive (+) cells were quantified. (B) The relative protein levels of Ki67 and PCNA were detected by western blot. Quantitation of signal intensities was performed using ImageJ software. (C) The invasion ability of HepG2 cells was detected by Transwell assay. HepG2 cells were stained with hematoxylin. (Magnification 40x). (D) The migration ability of HepG2 cells was detected by wound healing assay. (E) Epithelial mesenchymal transition (EMT) assay. $(\mathrm{F}, \mathrm{G})$ The relative protein levels of E-cadherin and $\mathrm{N}$-cadherin were detected by western blot. The signal intensities were quantitated by ImageJ software. *, $\mathrm{P}<0.05$, compared with the control group; ${ }^{*}, \mathrm{P}<0.05$, compared with the miR-377 inhibitor group; ${ }^{*}$, $\mathrm{P}<0.01$, compared with the control group; ${ }^{\prime \prime}, \mathrm{P}<0.01$, compared with the miR-377 inhibitor group. HULC, highly upregulated in liver cancer. 
group compared with miR-377-5p inhibitor (Figure $4 B$, $\mathrm{P}<0.05)$. Additionally, HepG2 cells invasion ability was promoted in the miR-377-5p inhibitor group in comparison to the control group, but HepG2 cells invasion ability was inhibited in the sh-HULC + inhibitor group in comparison to the miR-377-5p inhibitor group (Figure $4 C, \mathrm{P}<0.05$ ). HepG2 cells migration ability was induced in the miR-377$5 \mathrm{p}$ inhibitor group in comparison to the control group, but HepG2 cells migration ability was suppressed in the shHULC + inhibitor group in comparison to the miR-377$5 \mathrm{p}$ inhibitor group (Figure $4 D, E, \mathrm{P}<0.05$ ). Meanwhile, the $\mathrm{E}$-cadherin protein expression levels were increased by shHULC but decreased by miR-377-5p inhibitor $(\mathrm{P}<0.05)$. Besides, the $\mathrm{N}$-cadherin protein expression levels were decreased with sh-HULC but increased with miR-377-5p inhibitor $(\mathrm{P}<0.05$, Figure $4 F, G)$. Our findings suggested that down-regulation of HULC might suppress HepG2 cells proliferation and invasion abilities via derepression of miR$377-5 \mathrm{p}$.

\section{Sh-HULC inbibited tumor growth in vivo}

In comparison to the control group, the weight of the tumors from the mice were markedly suppressed by shHULC in vivo $(\mathrm{P}<0.05$, Figure $5 A)$. The relative mRNA leves of sh-HULC were reduced, while the miR-377$5 \mathrm{p}$ levels were expectedly elevated $(\mathrm{P}<0.05$, Figure $5 B)$. Figure $5 C$ showed the survival rate of mice with tumors. Interestingly, the survival rate of the sh-HULC group was higher than that of the control group $(\mathrm{P}<0.05)$. The result of immunohistochemistry clearly demonstrated that shHULC reduced the protein levels of Ki67 and N-cadherin in the tumor tissues of the mice $(\mathrm{P}<0.05$, Figure $5 D)$. These results indicated that down-regulation of HULC could inhibit tumor growth in nude mice.

\section{Discussion}

This study confirmed that the mRNA relative expressive levels of HULC were up-regulated in HCC cells, while the expression levels of miR-377-5p were down-regulated. Interestingly, we identified that HULC could contribute to the proliferation and invasion of HepG2 cells via sponging miR-377-5p, while down-regulation of HULC could inhibit the proliferation and invasion of HepG2 cells by derepressing miR-377-5p.

LncRNA HULC is an oncogene in numerous malignancies (7). Several studies have explored the tumorigenic function of HULC in HCC (16-18). According to these studies, in HCC, HULC accelerates metastasis and tumorigenesis through enhancing proliferation and epithelial-mesenchymal transition. Down-regulation of HULC improves the sensitivity of prostate cancer cells to irradiation in vivo and in vitro. HULC suppresses Beclin-1 phosphorylation, thus reducing autophagy, via the mTOR pathway (19). Knockdown of HULC inhibits pituitary adenoma GH3 cells via the up-regulation of miR-130b, the down-regulation of Forkhead box M1 (FOXM1), and the activation of the phosphoinositide-3kinase/serine-threonine kinase Akt/mammalian target of rapamycin (PI3K/Akt/mTOR) and Janus kinase-1 (JAK1)/ signal transducer and activator of transcription-3 (STAT3) pathways (20). These results demonstrated that HULC knockdown inhibited HepG2 cells proliferation and invasion abilities in vitro, as well as tumor growth in vivo, and promoted the survival of the mice.

A growing bank of evidence have demonstrated miR377 to be a tumor suppressor $(12,13,21)$. MiR-377-5p has been reported to inhibit cell proliferation, invasion, and cell cycle progression in lung cancer (22). MiR377 has been proved to suppress cell proliferation and invasion in HCC (13). HULC activates PI3 K/Akt/ mTOR pathway through inhibiting phosphatase and tensin homolog (PTEN) in human liver cancer cells (23). HULC accelerates the progress of breast cancer through regulating the expression of Ly-6/PLAUR domaincontaining 1 (LYPD1) by sponging miR-6754-5p (24). Overexpression of HULC induces the development of ovarian carcinoma by activating the PI3K/AKT/mTOR signaling pathway through suppressing the levels of miR$125 a-3 p$ (25). In our study, miR-377-5p downregulation were observed in HCC cells. Notably, overexpression of miR-377-5p suppressed cell proliferation and invasion in HCC. Interestingly, miR-377-5p was targeted by HULC, thus the regulatory activity of HULC in HCC was performed by sponging miR-377-5p.

The many candidate downstream genes of miR377-5p include T-lymphoma invasion and metastasis 1 (TIAM1) (13), Bcl-xL (12), DNA methyltransferase 1 (DNMT1) (21), and protein kinase B (AKT1) (22), each of which has been studied. We chose hypoxia-inhibitory factor- $1 \alpha$ (HIF-1 $\alpha$ ) (one of the target sites predicted by TargetScan), detected the expression, and verified the target relationship of miR-377-5p with HIF- $1 \alpha$. HIF$1 \alpha$ is a crucial transcription factor that contributes to the tumor EMT, which is characterized by the loss of cell 

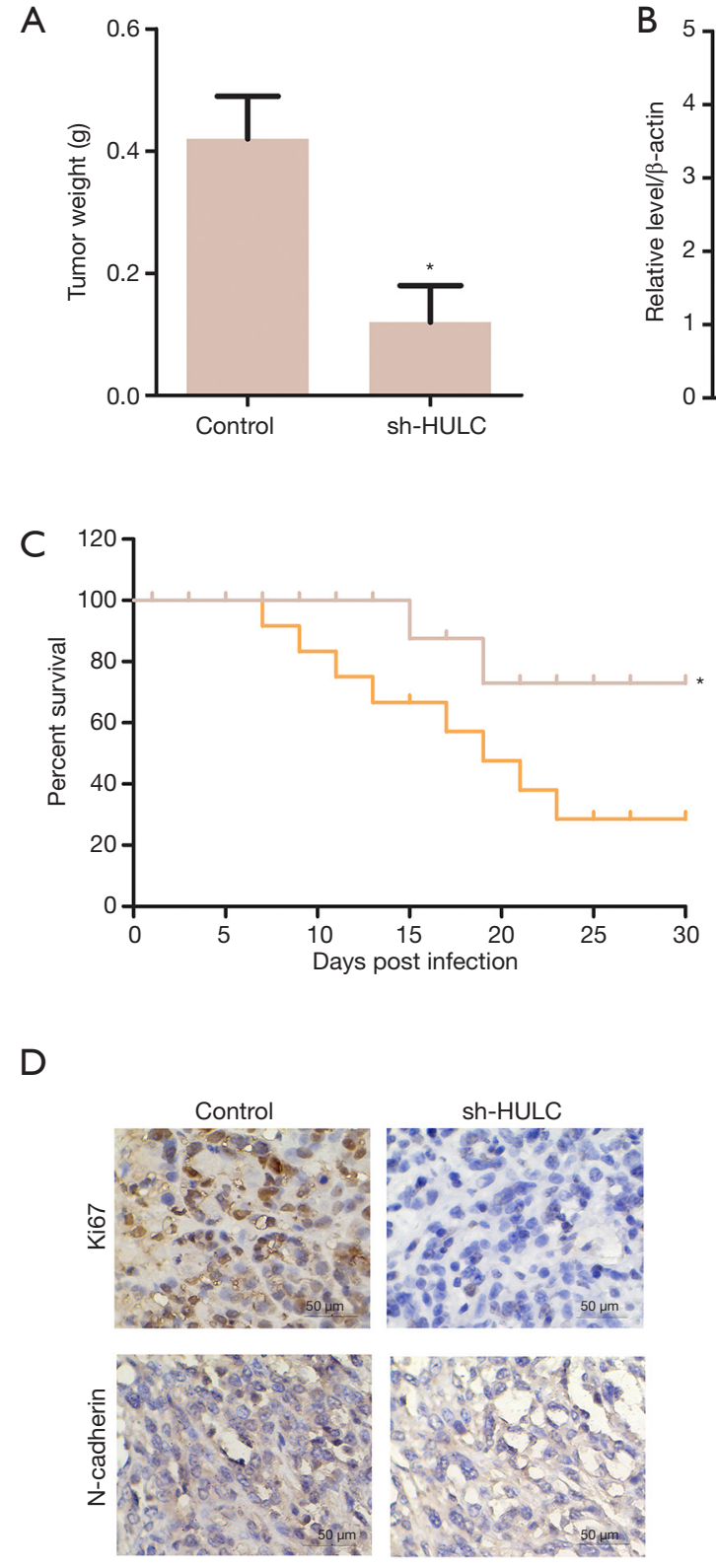

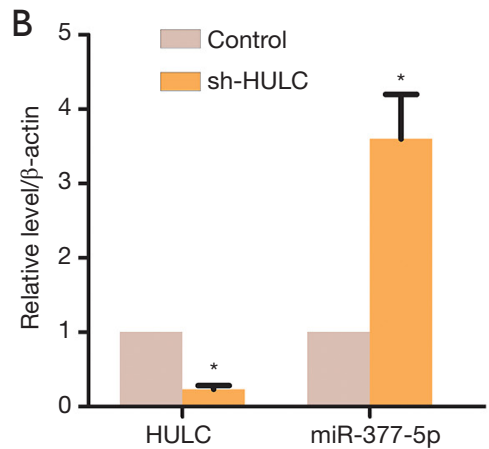

$$
\begin{aligned}
& + \text { Control } \\
& + \text { sh-HULC }
\end{aligned}
$$


acid (lincRNA)-p21 can significantly down-regulate the level of HIF-1 $\alpha$, thus downregulating the expression of VEGF and affecting the cell proliferation, apoptosis and migration in HCC (30). HIF-1 $\alpha$ plays an important role in the development of HCC by promoting HCC metastasis, epithelial mesenchymal transition (EMT) and vasculogenic mimicry (VM) through up-regulating LOXL2 (31). HIF-1 $\alpha$ promotes HCC cell migration and invasion by modulating IL-8 via the NF- $\mathrm{KB}$ pathway (32). As a result (see the Supplementary materials), the mRNA and protein levels of HIF $1 \alpha$ were reduced by HULC knockdown. These results indicated that HIF-1 $\alpha$ might be a target of miR-377-5p, and HULC enhanced the growth and invasion of HepG2 cells by sponging miR-377-5p from HIF- $1 \alpha$.

\section{Conclusions}

In summary, this study showed that down-regulation of HULC inhibited the proliferation and invasion of HepG2 cells. Meanwhile, down-regulation of HULC had an inhibitory effect on tumor growth in vivo. In vitro and in vivo experiments confirmed that HULC is involved in the progression of HCC and the underlying mechanism may be through the miR-377-5p/HIF $1 \alpha$ pathway. The result of our experiments also presented the HULC/miR-377-5p/HIF1 $\alpha$ axis as a promising therapeutic target in HCC.

\section{Acknowledgments}

Funding: None.

\section{Footnote}

Reporting Checklist: The authors have completed the ARRIVE reporting checklist. Available at http://dx.doi. org/10.21037/atm-20-5556

Data Sharing Statement: Available at http://dx.doi. org/10.21037/atm-20-5556

Conflicts of Interest: All authors have completed the ICMJE uniform disclosure form (available at http://dx.doi. org/10.21037/atm-20-5556). The authors have no conflicts of interest to declare.

Ethical Statement: The authors are accountable for all aspects of the work in ensuring that questions related to the accuracy or integrity of any part of the work are appropriately investigated and resolved. All animal experiments in this study received approval from the Committee for Animal Experiments of the First Affiliated Hospital of Henan University. This study also conformed to the NIH guidelines.

Open Access Statement: This is an Open Access article distributed in accordance with the Creative Commons Attribution-NonCommercial-NoDerivs 4.0 International License (CC BY-NC-ND 4.0), which permits the noncommercial replication and distribution of the article with the strict proviso that no changes or edits are made and the original work is properly cited (including links to both the formal publication through the relevant DOI and the license). See: https://creativecommons.org/licenses/by-nc-nd/4.0/.

\section{References}

1. Siegel RL, Miller KD, Jemal A.Cancer statistics, 2018. CA Cancer J Clin 2018;68:7-30.

2. Bae SH, Park HC. Local modalities for inoperable hepatocellular carcinoma: radiofrequency ablation versus stereotactic body radiotherapy. Ann Transl Med 2018;6:S3.

3. Hu C, Cui S, Zheng J, et al. MiR-875-5p inhibits hepatocellular carcinoma cell proliferation and migration by repressing astrocyte elevated gene-1 (AEG-1) expression. Transl Cancer Res 2018;7:158-69.

4. Peng WX, Koirala P, Mo YY. LncRNA-mediated regulation of cell signaling in cancer. Oncogene 2017;36:5661-7.

5. Abbastabar M, Sarfi M, Golestani A, et al. lncRNA involvement in hepatocellular carcinoma metastasis and prognosis. EXCLI J 2018;17:900-13.

6. Chen S, Wu DD, Sang XB, et al. The lncRNA HULC functions as an oncogene by targeting ATG7 and ITGB1 in epithelial ovarian carcinoma. Cell Death Dis 2017;8:e3118.

7. Yu X, Zheng H, Chan MT, et al. HULC: an oncogenic long non-coding RNA in human cancer. J Cell Mol Med 2017;21:410-7.

8. Ou ZL, Luo Z, Lu YB. Long non-coding RNA HULC as a diagnostic and prognostic marker of pancreatic cancer. World J Gastroenterol 2019;25:6728-42.

9. Chen C, Wang K, Wang Q, et al. LncRNA HULC mediates radioresistance via autophagy in prostate cancer cells. Braz J Med Biol Res 2018;51:e7080.

10. Abba M, Mudduluru G, Allgayer H. MicroRNAs in 
cancer: small molecules, big chances. Anticancer Agents Med Chem 2012;12:733-43.

11. Anwar SL, Lehmann U. MicroRNAs: Emerging Novel Clinical Biomarkers for Hepatocellular Carcinomas. J Clin Med 2015;4:1631-50.

12. Ge H, Zou D, Wang Y, et al. MicroRNA-377 Downregulates Bcl-xL and Increases Apoptosis in Hepatocellular Carcinoma Cells. Oncol Res 2017;25:29-34.

13. Chen G, Lu L, Liu C, et al. MicroRNA-377 suppresses cell proliferation and invasion by inhibiting TIAM1 expression in hepatocellular carcinoma. PLoS One 2015;10:e0117714.

14. Sun Y, Zheng ZP, Li H, et al. ANRIL is associated with the survival rate of patients with colorectal cancer, and affects cell migration and invasion in vitro. Mol Med Rep 2016;14:1714-20.

15. Zhang W, Che Q, Tan H, et al. Marine Streptomyces sp. derived antimycin analogues suppress HeLa cells via depletion HPV E6/E7 mediated by ROS-dependent ubiquitin-proteasome system. Sci Rep 2017;7:42180.

16. Wang Y, Chen F, Zhao M, et al. The long noncoding RNA HULC promotes liver cancer by increasing the expression of the HMGA2 oncogene via sequestration of the microRNA-186. J Biol Chem 2017;292:15395-407.

17. Xiong H, Li B, He J, et al. lncRNA HULC promotes the growth of hepatocellular carcinoma cells via stabilizing COX-2 protein. Biochem Biophys Res Commun 2017;490:693-9.

18. Xiong H, Ni Z, He J, et al. LncRNA HULC triggers autophagy via stabilizing Sirt1 and attenuates the chemosensitivity of HCC cells. Oncogene 2017;36:3528-40.

19. Chen C, Wang K, Wang Q, et al. LncRNA HULC mediates radioresistance via autophagy in prostate cancer cells. Braz J Med Biol Res 2018;51:e7080.

20. Rui QH, Ma JB, Liao YF, et al. Effect of lncRNA HULC knockdown on rat secreting pituitary adenoma GH3 cells. Braz J Med Biol Res 2019;52:e7728.

21. Azizi M, Fard-Esfahani P, Mahmoodzadeh H, et al. MiR377 reverses cancerous phenotypes of pancreatic cells via suppressing DNMT1 and demethylating tumor suppressor genes. Epigenomics 2017;9:1059-75.

22. Wu H, Liu HY, Liu WJ, et al. miR-377-5p inhibits lung cancer cell proliferation, invasion, and cell cycle progression by targeting AKT1 signaling. J Cell Biochem
2018. [Epub ahead of print].

23. Xin X, Wu M, Meng Q, et al. Long noncoding RNA HULC accelerates liver cancer by inhibiting PTEN via autophagy cooperation to miR15a. Mol Cancer 2018;17:94.

24. Wang N, Zhong C, Fu M, et al. Long Non-Coding RNA HULC Promotes the Development of Breast Cancer Through Regulating LYPD1 Expression by Sponging miR-6754-5p. Onco Targets Ther 2019;12:10671-9.

25. Chu P, Xu L, Su H. HULC functions as an oncogene in ovarian carcinoma cells by negatively modulating miR125a-3p. J Physiol Biochem 2019;75:163-71.

26. Azab AK, Hu J, Quang P, et al. Hypoxia promotes dissemination of multiple myeloma through acquisition of epithelial to mesenchymal transition-like features. Blood 2012;119:5782-94.

27. Xiang ZL, Zeng ZC, Fan J, et al. The expression of HIF-1alpha in primary hepatocellular carcinoma and its correlation with radiotherapy response and clinical outcome. Mol Biol Rep 2012;39:2021-9.

28. Lin D, Wu J. Hypoxia inducible factor in hepatocellular carcinoma: A therapeutic target. World J Gastroenterol 2015;21:12171-8.

29. Song Z, Liu T, Chen J, et al. HIF-1 $\alpha$-induced RIT1 promotes liver cancer growth and metastasis and its deficiency increases sensitivity to sorafenib. Cancer Lett 2019;460:96-107.

30. Ye Y, Peng Y, Li Y, et al. Effect of lincRNA-p21 targeting HIF-1 $\alpha$ on biological functions of liver cancer cells. Oncol Lett 2019;17:4964-8.

31. Wang M, Zhao X, Zhu D, et al. HIF-1 $\alpha$ promoted vasculogenic mimicry formation in hepatocellular carcinoma through LOXL2 up-regulation in hypoxic tumor microenvironment. J Exp Clin Cancer Res 2017;36:60.

32. Feng $W$, Xue $T$, Huang $S$, et al. HIF-1 $\alpha$ promotes the migration and invasion of hepatocellular carcinoma cells

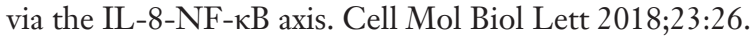

(English Language Editor: J. Reynolds)

Cite this article as: Yan C, Wei S, Han D, Wu L, Tan L, Wang H, Dong Y, Hua J, Yang W. LncRNA HULC shRNA disinhibits miR-377-5p to suppress the growth and invasion of hepatocellular carcinoma in vitro and hepatocarcinogenesis in vivo. Ann Transl Med 2020;8(20):1294. doi: 10.21037/atm-20-5556 
A

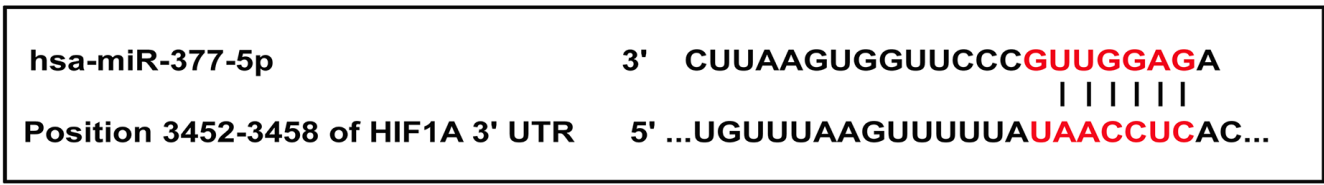

B

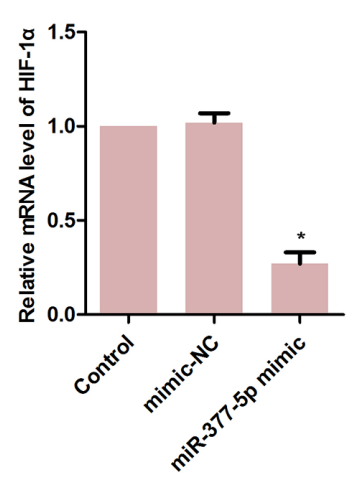

E

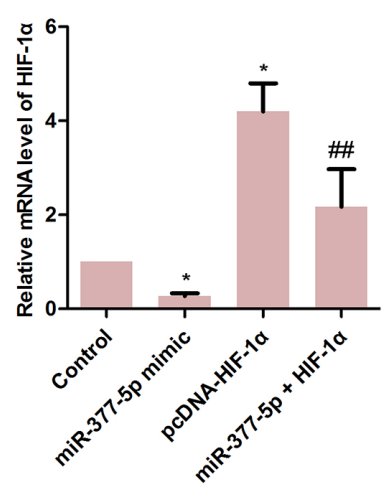

G

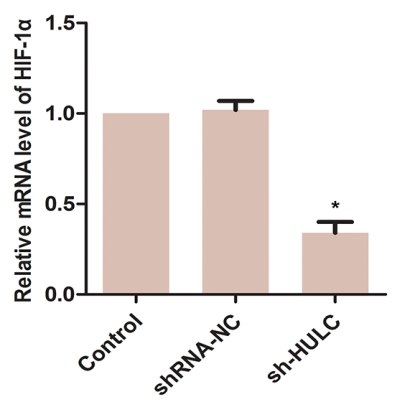

C

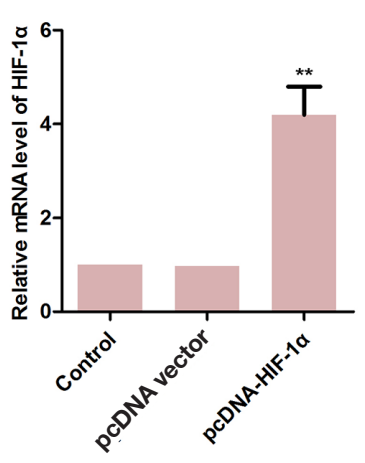

F

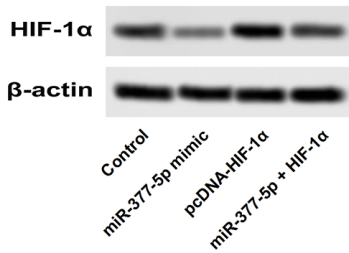

$\mathrm{H}$

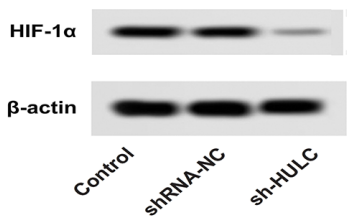

D
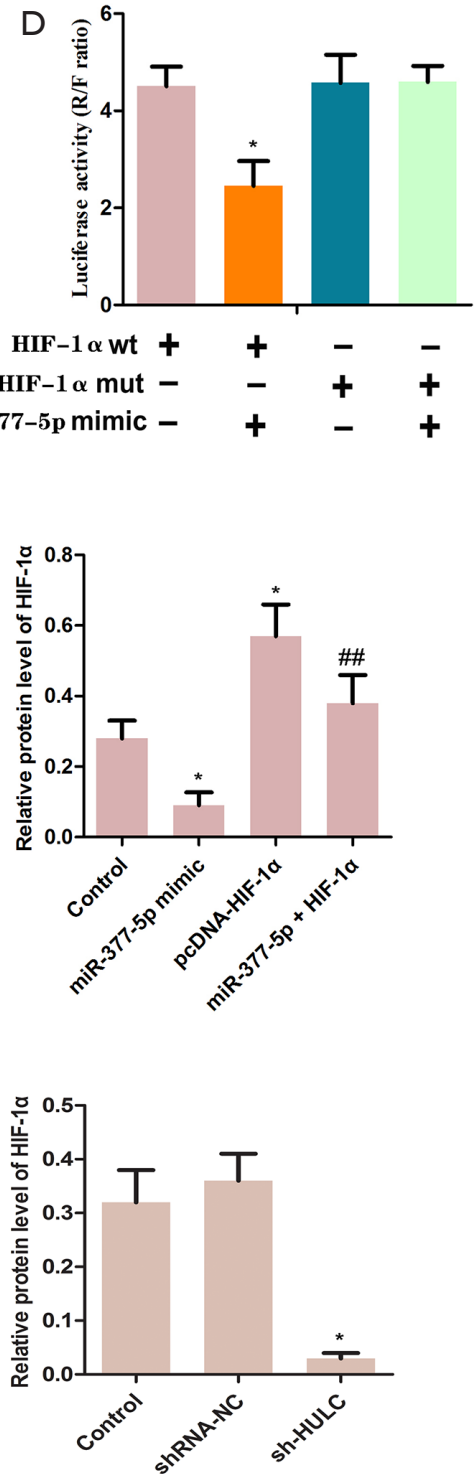

Figure S1 The target relationship between HIF-1 $\alpha$ and miR-377-5p. (A) Predicted binding sites between HIF-1 $\alpha$ and miR-377-5p. (B) The mRNA levels of HIF-1 $\alpha$ were detected by qRT-PCR in control group, mimic-NC group and miR-377-5p mimic group. (C) The mRNA levels of HIF-1 $\alpha$ were detected by qRT-PCR in control group, pcDNA-vector group and pcDNA-HIF-1 $\alpha$ group. (D) Luciferase activity was presented relative to that of the control and mutant or wild-type HIF-1 $\alpha$ 3'UTR. (E) The mRNA levels of HIF-1 $\alpha$ were detected by qRT-PCR in control group, miR-377-5p mimic group, pcDNA-HIF-1 $\alpha$ group and miR-377-5p + HIF-1 $\alpha$ group. (F) The relative protein levels of HIF-1 $\alpha$ were detected by western blotting in control group, miR-377-5p mimic group, pcDNA-HIF-1 $\alpha$ group and miR-377-5p + HIF-1 $\alpha$ group. (G) The mRNA levels of HIF-1 $\alpha$ were detected by qRT-PCR in control group, shRNA-NC group and sh-HULC group. $(\mathrm{H})$ The relative protein levels of HIF-1 $\alpha$ were detected by western blotting in control group, shRNA-NC group and sh-HULC group. ${ }^{*}, \mathrm{P}<0.05$, compared with the control group; ${ }^{* *}, \mathrm{P}<0.01$, compared with the control group; ${ }^{\text {\#\# }}, \mathrm{P}<0.01$, compared with the pcDNA-HIF- $1 \alpha$ group). qRT-PCR, quantitative real-time polymerase chain reaction. 